\title{
Cosmic infrared background and early stellar populations
}

\author{
A. Kashlinsky \\ SSAI and Code 665, Observational Cosmology Laboratory, Goddard Space Flight \\ Center, Greenbelt, MD 20771 \\ Email: kashlinsky@stars.gsfc.nasa.gov
}

\begin{abstract}
Cosmic infrared background (CIB) contains information about galaxy luminosities over the entire history of the Universe and can be a powerful diagnostic of the early populations otherwise inaccessible to telescopic studies. Its measurements are very difficult because of the strong IR foregrounds from the Solar system and the Galaxy. Nevertheless, substantial recent progress in measuring the CIB and its structure has been made. The measurements now allow to set significant constraints on early galaxy evolution and, perhaps, even detect the elusive Population III era. We discuss briefly the theory behind the CIB, review the latest measurements of the CIB and its structure, and discuss their implications for detecting and/or constraining the first stars and their epochs.
\end{abstract}

Key words: Cosmic infrared background, population III stars, reionization, galaxy formation, galaxy evolution

\section{Introduction}

Cosmic infrared background (CIB) is a repository of stellar emissions throughout the entire history of the Universe. Cosmic expansion shifts any photon emitted in the visible/UV bands at high $z$ into the near-IR (NIR) and the high- $z$ NIR photons will today appear in mid- to far-IR. Consequently, the NIR part of the CIB spectrum $(1 \mu \mathrm{m}<\lambda<10 \mu \mathrm{m})$ probes the history of direct stellar emissions from the early Universe, and the longer wavelengths contain information about the early dust production and evolution.

The last several years have seen significant progress in CIB studies, both in identifying/constraining its mean level (isotropic component) and fluctuations 
(see (12) for a recent review). CIB contains emissions also from objects inaccessible to current (or even future) telescopic studies and can, therefore, provide unique information on the history of the Universe at very early times. One particular example of such objects, which is of prime relevance to this meeting, are Population III, the so-far hypothetical zero-metallicity stars expected to have preceded normal stellar populations seen in the farthest galaxies to-date.

This review is organized as follows: we briefly present theoretical basis behind the CIB in Sec. 2, and discuss the two broad classes of contributors in Sec. 3. In Sec. 4 we review the old (pre-Spitzer) measurements of the CIB and Sec. 5 gives an overview of the very recent results that were obtained from Spitzer IRAC deep exposures by our team (13). We conclude in Sec. 6.

\section{Theory}

The total CIB flux, $F \equiv \lambda I_{\lambda}$, produced in $d z$ by a population with comoving luminosity function $n(L)$ and luminosity density $\mathcal{L}(z)=\int n(L) L d L$ is:

$$
\frac{d F}{d z}=\frac{c H_{0}^{-1}}{4 \pi} \frac{1}{(1+z)^{2}} \frac{d\left(H_{0} t\right)}{d z} \sum_{i} \mathcal{L}(z)\left[\lambda f_{\lambda}\left(\frac{\lambda}{1+z} ; z\right)\right]
$$

Here $f_{\lambda}$ is the SED of the sources/galaxies normalized to $\int f_{\lambda} d \lambda=1$.

Because the emitters are clustered, the CIB produced by them will have fluctuations reflecting the underlying clustering with power spectrum $P_{3}(k)$. The flux fluctuation is $\delta F=F-\langle F\rangle$ and in the limit of small angles $(<1 \mathrm{sr})$ a cartesian geometry can be used in its Fourier expansion, $\delta F(\vec{\theta})=\sum f_{q} \exp (-i \vec{q} \cdot \vec{\theta})$. The 2-D power spectrum of the CIB is $P_{2}(q)=\left\langle\left|f_{q}\right|^{2}\right\rangle$ and is related to $P_{3}$ via the Limber equation. The rms fluctuations on scale encompassing angular wavelength $2 \pi / q$ is $\delta F_{\text {rms }} \simeq \sqrt{q^{2} P_{2} / 2 \pi}$. In order to connect the measured/projected power spectrum to the underlying 3-D distribution, it is convenient to use the Limber equation in the form (12):

$$
\frac{q^{2} P_{2}(q)}{2 \pi}=\pi t_{0} \int\left(\frac{d I_{\nu^{\prime}}}{d t}\right)^{2} \Delta^{2}\left(q d_{A}^{-1} ; z\right) d t
$$

where $t_{0}$ is the time-length of the period over which the CIB is produced and

$$
\Delta^{2}(k)=\frac{1}{2 \pi^{2}} \frac{k^{2} P_{3}(k)}{c t_{0}}
$$


is the mean-squared fluctuation in number of sources within a line-of-sight cylinder of volume $k^{-2} c t_{0}$. Eqs. 2,3 can be rewritten in terms of the total CIB flux as

$$
\delta F\left(\frac{2 \pi}{q}\right) \equiv \sqrt{\frac{q^{2} P_{2}(q)}{2 \pi}}=F \Delta\left(q d_{A}^{-1}(\bar{z})\right)
$$

where $\bar{z}$ is a suitably averaged effective redshift of the populations contributing to CIB fluctuations.

In addition to fluctuations produced by clustering, there will also be a shot noise component due to Poissonian fluctuations from sources occasionally entering the beam. This component is small for surveys with large beams, which contain many sources, but can be important for $\sim \operatorname{arcsec}$ or better resolution surveys. The power spectrum of the shot noise contribution to the CIB from sources with angular density $d N(m) / d m$ per magnitude range $d m$ is:

$$
P_{\mathrm{SN}}=\int f^{2}(m) \frac{d N}{d m} d m
$$

where $f(m) \equiv f_{0} 10^{-0.4 m}$ is the flux corresponding to magnitude $m$.

Any measurement of the CIB power spectrum is affected by the sample or cosmic variance. This is a consequence of the fact that one is trying to deduce the power spectrum of an infinite distribution from a field of finite extent. The uncertainty is $N_{q}^{-1 / 2}$ where $N_{q}$ is the number of independent elements in Fourier space that goes into determining the power at given $q$. Because at large scales (small $q$ ) there are progressively fewer such elements, reaching just one at half the field extent, in order to reliably probe fluctuations on scale $\theta$ one needs a field-of-view a few times larger.

In surveys with high angular resolution it becomes possible to remove bright(er) more nearby galaxies. In this way one can isolate the contributions to the CIB from fainter galaxies located at progressively higher $z(16 ; 22)$.

\section{CIB contributors}

Galactic and Solar System foregrounds are the major obstacles to space-based measurements of the CIB. Galactic stars are the main contributors at near-IR ( $<$ a few micron), zodiacal light from the dust in the Solar system contributes mostly between $\sim 10$ and $\sim 50$ micron, and Galactic cirrus emission produces most of the foreground at IR wavelengths longward of $\sim 50$ micron. Accurately removing the foregrounds presents a challenge and many schemes have 
been developed to do this as well as possible. Stars can be removed in surveys with fine angular resolution $(10 ; 16 ; 22)$ or by statistically extrapolating the various stellar contributions to zero $(15 ; 29)$. Zodiacal light contributions usually are removed using the DIRBE zodi model $(18)$ or its derivatives $(10 ; 20)$. Galactic cirrus and zodiacal light are both intrinsically diffuse, but are fairly homogeneous adding to the usefulness of CIB fluctuations studies at mid- to far-IR (14).

For purposes of this review we divide the extragalactic CIB contributors into two groups: 1) "ordinary" galaxies, which are metal-enriched systems of normal stellar populations with Salpeter of Scalo IMFs, and 2) Population III stars that preceded ordinary galaxy populations. The first category, which we term ordinary galaxies contribute around $\sim 1-10 \mathrm{nW} / \mathrm{m}^{2} / \mathrm{sr}$ to the mean CIB in the near- to mid-IR. At many wavelengths their total flux can be measured by summing up contributions from deep galaxy counts, $\int f(m) \frac{d N}{d m} d m$, and several surveys go sufficiently deep to observe this quantity saturate. In this way one can arrive at a fairly robust estimate of the total contribution of the ordinary galaxies to the CIB. Such contributions in near-IR are shown in Fig. 1 with open squares for HDF data (19) and with open diamonds for Spitzer/IRAC deep counts (7).

Population III are currently believed to have been very massive stars, as discussed by Tom Abel in these proceedings, but even then their contributions can only be estimated in a theoretical, and necessarily speculative, manner. However, if the CIB measurements are accurate and the net contribution from ordinary galaxies is known to a reasonable accuracy, the difference between the two is likely to originate from Population III stars located at very early cosmic times ( $z>10$ or so). Even the most careful measurements of the CIB mean levels can be significantly affected by the systematics and be mistaken for the various residual errors. On the other hand, Population III stars, whose emission arises at epochs when the spatial spectrum of galaxy clustering is not yet evolved, should have produced a unique and measurable signature in the near-IR CIB fluctuations $(4 ; 17)$. That signature, both its spectrum in the angular and energy frequency domain, could provide - if and when measured the ultimate insight into the Population III epochs. There are several intuitive reasons why Population III produce significant CIB levels, both its mean and fluctuations:

- Each unit mass of Population III (if made of massive stars) emits $\sim 10^{5}$ more light than normal stars and massive stars (assuming Population III were indeed massive stars) radiate with a higher mean radiative efficiency, $\epsilon=0.007$, than the present day stellar populations, leading to substantial net flux.

- Because their era was presumably brief, Population III epochs contain less projected volume than the ordinary galaxy populations spanning the epochs of Population I and I stars. Hence, the larger relative fluctuations.

- Biasing is higher for Population III because they form out of rarer regions 
which leads to the amplified correlations.

\section{4 "Old" results: DIRBE, FIRAS, IRTS, 2MASS}

The current best direct measurements of the mean level of the CIB come from the data obtained with the COBE DIRBE and FIRAS instruments and the IRTS mission. The current measurements of the CIB anisotropies come from the above missions, as well as the ground based deep 2MASS images and the very latest Spizter results. The latter are discussed separately in the next section.

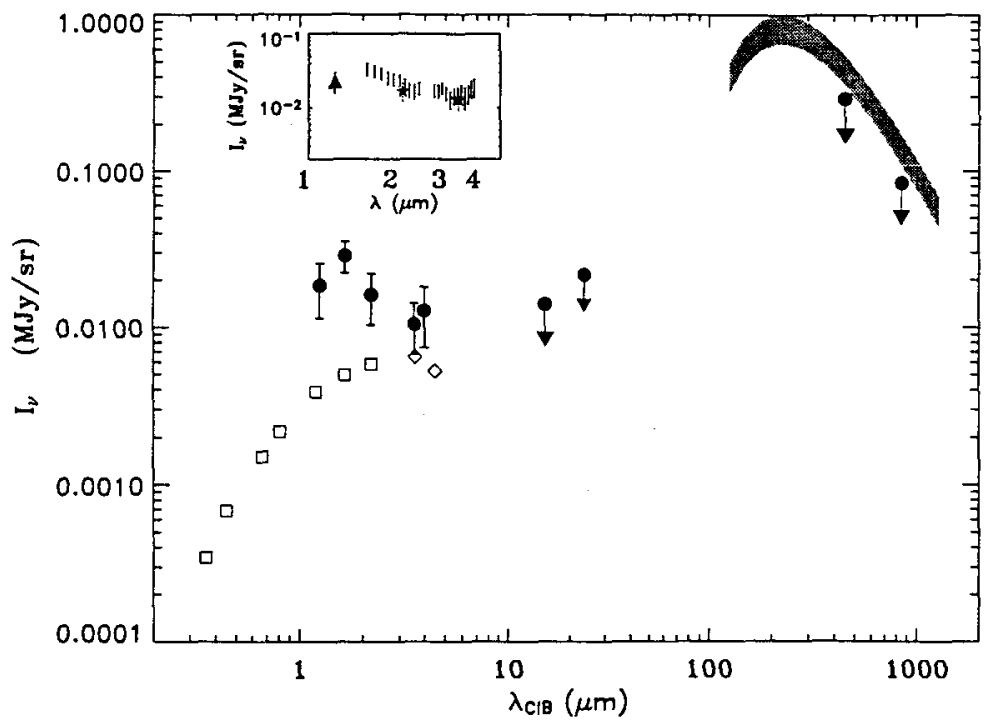

Figure 1: Inset shows the near-IR CIB detections with their error bars: filled triangle is from (5) based on DIRBE data, vertical error bars from IRTS data (20), and asterisks are from analyses based on DIRBE data $(6 ; 10 ; 29)$. Shaded region corresponds to the far-IR CIB based on FIRAS data (24) with a fit and uncertinties from (8). Open symbols at visible to near-IR correspond to net contributions from ordinary galaxies: those derived from the HST data are shown with squares (19) and those from Spitzer data are shown with diamonds (7). The difference is the CIB excess over contributions from ordinary galaxies which is shown with filled circles marked with downward pointing arrows where galaxies can account, within errors, for the CIB and only upper limits (essentially errors) can be claimed (12). 
In the near-IR detections are difficult because of the substantial foreground by Galactic stars. Claims of detections of the mean isotropic part of the CIB are based on various analyses of DIRBE and IRTS data $(6 ; 20 ; 10 ; 29 ; 5 ; 1)$. The measurements agree with each other, although the methods of analysis and foreground removal differ substantially. They also agree with the measured amplitude of CIB fluctuations discussed later in this section. The results seem to indicate fluxes significantly in excess of those from observed galaxy populations. The near-IR detections of the CIB are summarized in the inset of Fig. 1. Total fluxes from galaxies in NIR from HST and Spitzer IRAC measurements are shown with open symbols in Fig. 1; they saturate at Vega magnitude of about $\mathrm{K} \simeq 20-22$ and are a factor is $\sim 2-3$ below the detected CIB. The difference between the claimed CIB levels and the total fluxes from "ordinary" (i.e. not Population III) galaxies are shown with their uncertainties as filled circles in Fig. 1.

In mid-IR there are no CIB detections, but the best upper limits come from gamma-ray absorption limits derived from spectra of nearby $(z \sim 0.1)$ blazars and are quite low $\sim 4-5 \mathrm{nw} / \mathrm{m}^{2} / \mathrm{sr}(28)$. Because the foregrounds are very homogeneous at these wavelengths, one obtains slightly higher, but comparable, CIB upper limits $\left({ }_{\sim}^{<} 10 \mathrm{nW} / \mathrm{m}^{2} / \mathrm{sr}\right)$ from the fluctuations analysis (14). The best upper limits on the CIB at mid-IR are comparable to the net contributions from ISO (21) and Spitzer MIPS (23) galaxy counts of $\simeq 3 \mathrm{nW} / \mathrm{m}^{2} / \mathrm{sr}$ at 15 and $24 \mu \mathrm{m}$.

Far-IR CIB measurements come from the COBE FIRAS $(24 ; 8)$ and COBE DIRBE $(27 ; 11)$ data analysis. They are broadly consistent although the COBE DIRBE detections at 140 and $240 \mu \mathrm{m}$ give larger fluxes. The fit to the FIRAS CIB detection of (8) is shown within its uncertainties as the shaded region in Fig. 1. The far-IR CIB is expected to originate from dust (re)emissions and the shape of the FIRAS detection in Fig. 1 confirms this. The total galaxy fluxes there come from SCUBA galaxies (3) and, within the uncertainties, they can account for the CIB at these wavelengths.

Detections of CIB fluctuations come from three independent experiments and are consistent with each other if accounting is made for contributions from removed galaxies, such as in deep 2MASS data (22). The DIRBE data based analysis, extrapolating the contribution from Galactic stars to 0 , gives a statistically significant CIB fluctuation at $\sim 0.5^{\circ}$ in the DIRBE first 4 channels or $1.25,2.2,3.5$ and $4.5 \mu \mathrm{m}$ (15). The IRTS team (20) measured the spectrum of CIB anisotropies on degree scales from IRTS data band-averaged to $\sim 2 \mu \mathrm{m}$; their results are consistent with (15). Because the beam was large in both instruments, no galaxy removal was possible in the data and the CIB anisotropies arise from all galaxies, i.e. from $z=0$ to the earliest times. Based on the amplitude of the present-day galaxy clustering, the NIR detections exceed the expectations from galaxies evolving with no or little evolution by a 
factor of $\sim 2-3$ and are consistent with the measured (high) mean CIB levels in the NIR.

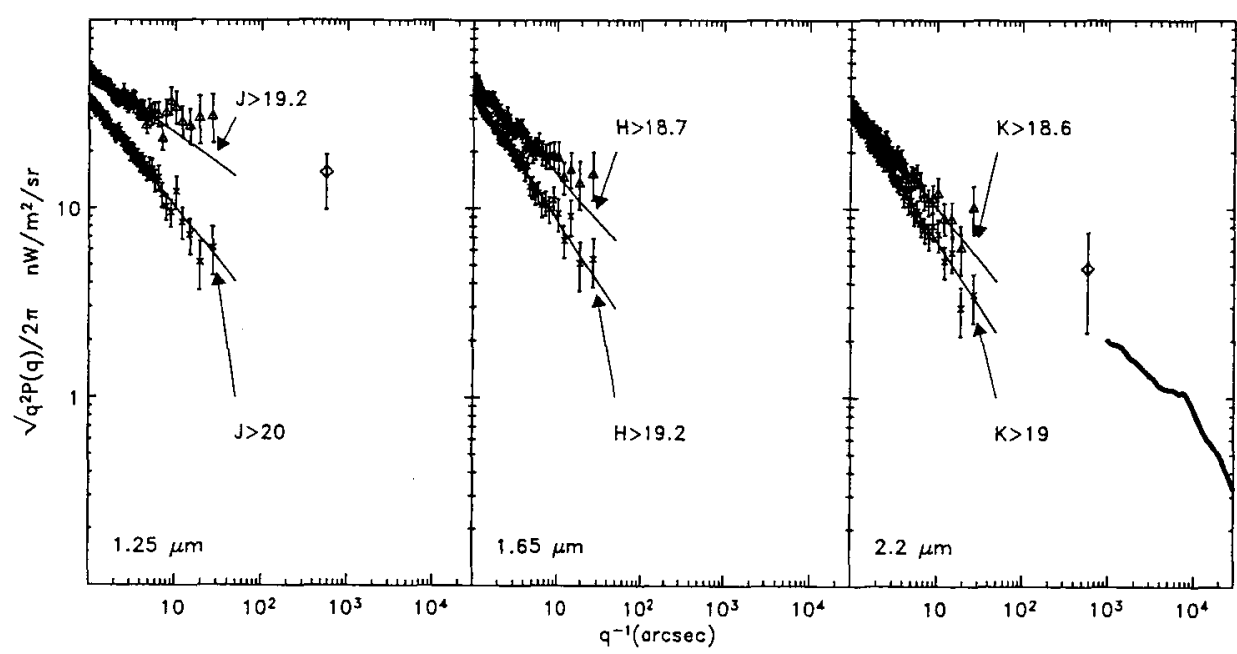

Figure 2: CIB fluctuations at $\mathrm{J}, \mathrm{H}$ and $\mathrm{K}$ bands: open triangles and crosses show CIB fluctuations arising from galaxies fainter than the limit marked near the least squares fit lines based on deep exposure 2MASS data $(16 ; 22)$. In the right panel for $2.2 \mu \mathrm{m}$ the open diamond with the $92 \%$ confidence level error shows detection based on DIRBE data from (15). The thick solid line shows the CIB fluctuation spectrum based on the IRTS data from (20). Both of the latter correspond to CIB from all galaxies; the low resolution of the DIRBE and IRTS/NIRS instruments did not allow for galaxy removal.

Analysis of deep 2MASS data $(16 ; 22)$ took the measurements of the CIB anisotropies one step further in that it has allowed to remove galaxies out to $K \simeq(18.5-19)$ and measure the spectrum of CIB anisotropies from fainter galaxies in the $\mathrm{J}, \mathrm{H}$, and $\mathrm{K}$ 2MASS bands on sub-arcminute angular scales. These galaxies are typically located at cosmological times when the Universe was less than $\sim$ half its present age so this measurement isolates CIB ansitropies arising from the emissions in the Universe at these early times. When extrapolated to the present-day the 2MASS based results are consistent with the DIRBE- and IRTS-based measurements. Fig. 2 summarizes the 2MASSbased results and compares them with the other measurements.

If one were to remove galaxies to fainter limits than was possible in the 2MASS data, one can probe emissions arising at still earlier cosmic times. There is a possibility - of direct relevance to the topics of this meeting - of Population III being responsible for the CIB excess $(26 ; 25)$. In order to directly measure such a contribution it is necessary to remove ordinary galaxies down to very faint flux limits. This was impossible to do with the old instruments, which 
either had low resolution and sensitivity (DIRBE and IRTS/NIRS) or were ground-based (2MASS) and affected by the atmosphere. We discuss a recent Spitzer-based measurement of the CIB from early epochs in the next section.

\section{New results from Spitzer IRAC deep images}

There is no direct observational evidence on what the first stars, Population III, were, although it is (now) generally believed that they had to be very massive stars. Recently, two teams have suggested that Population III, if massive, should have left substantial CIB fluctuations with a spectrum serving as their signature $(4 ; 17)$. Uncovering these anisotropies is a difficult and challenging task, but it offers a concrete observational test of and probe into the Population III era. We (Kashlinsky, Arendt, Mather, Moseley) have attempted to make a measurement of these CIB fluctuations in deep images obtained with Spitzer IRAC at 3.6, 4.5, 5.8 and $8 \mu \mathrm{m}$. This analysis is presented in a manuscript forthcoming in Nature (13) to which we refer readers for details; below is a brief summary of the steps in the measurement and the main results.

The main data came from the IRAC GTO observations with $\sim 8-9$ hour integration of a field located at sufficiently high Galactic latitude $\left(b_{\mathrm{Gal}}=36.1^{\circ}\right)$. Additionally, we analyzed shallower observations for 2 auxiliary fields in order to test for isotropy of any cosmological signal. The datasets were assembled out of the individual frames using the least calibration method of (9). This selfcalibration procedure has advantages over the standard pipeline calibration of the data in that the derived detector gain and offsets match the detector at the time of the observation, rather than at the time of the calibration observations. The most noticeable difference is that the different AORs are affected by different patterns of residual images left by prior observations, which if not properly removed, can leave artifacts in the final images (2).

For the final analysis we selected a subfield of $\simeq 5^{\prime} \times 10^{\prime}$ with a fairly homogeneous coverage. After the image assembly we removed a liner gradient from each map. Individual sources have been clipped iteratively as positive flux excursions above a fixed threshold. We used the full-array PSF in removing the residual extended parts of the identified sources by a CLEAN-type procedure. Fig. 3 shows the final image for Channel $1(3.6 \mu \mathrm{m})$ of IRAC. This image has $>75 \%$ pixels left which allows robust computation of the diffuse flux Fourier transforms, $f_{q}$. CIB fluctuations from Population III located at very high $z$ should be independent of the clipping threshold, so we also clipped progressively deeper to verify that our results are threshold-independent. As more pixels are removed, it becomes impossible to evaluate robust Fourier transforms, so we calculated instead the diffuse flux correlation function, which is related to the power spectrum by an integral transform. The power spectrum 
was evaluated as $P(q)=\left\langle|f(q)|^{2}\right\rangle$ where the average is taken over narrow concentric rings around a central $q$. Instrument noise was evaluated by dividing the data into two subsets $(A, B)$ containing the odd and even numbered frames and evaluating the power spectrum of the $\frac{1}{2}(A-B)$ map.

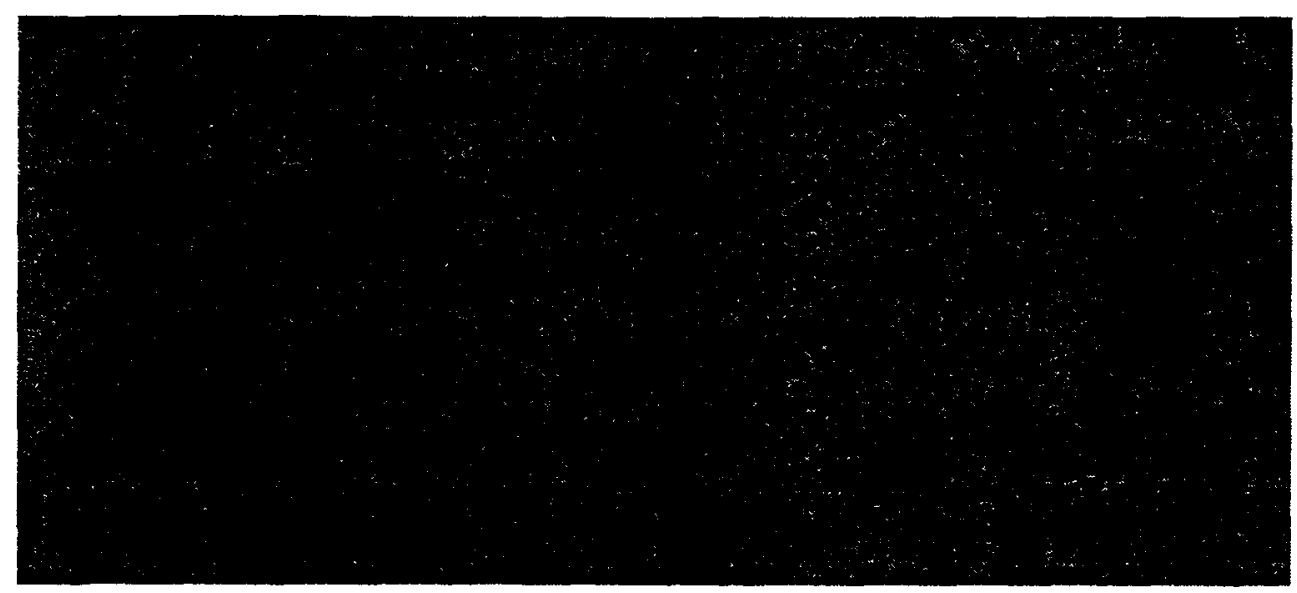

Figure 3 Final map for analysis from (13) at $3.6 \mu \mathrm{m}$. The map has point sources subtracted and clipped. This positive image is scaled from $[-2.0,4.0] \mathrm{nW} \mathrm{m} \mathrm{m}^{-2} \mathrm{sr}^{-1}$. The regions of clipped sources are indicated by black area which are set to 0.0 for further analysis.

In this analysis we detected fluctuations significantly exceeding the instrument noise. Fig. 4 shows the CIB fluctuation (the instrument noise has been subtracted) for the IRAC Channel 1 at $3.6 \mu \mathrm{m}$. The excess fluctuation on arcminute scales in the $3.6 \mu \mathrm{m}$ channel is $\sim 0.1 \mathrm{nW} / \mathrm{m}^{2} / \mathrm{sr}$; our analysis shows a similar amplitude in the longer IRAC bands indicating that the energy spectrum of the arcminute scale fluctuations is flat to slowly rising with increasing wavelength at least over the IRAC range of wavelengths.

The signal is significantly higher than the instrument noise and the various systematics effects we investigated cannot account for it. We find a statistically significant correlation between the channels for the region of overlap. We also find that the correlation function evaluated at deeper clipping cuts, when we have too few pixels left for Fourier analysis, remains the same and is consistent with the power spectrum evaluations. This suggests that the signal comes from the sky, which contains contributions from the remaining suspects: zodiacal emission, Galactic cirrus, and the extragalactic components: the "ordinary" galaxies and the putative Population III.

The zodiacal and Galactic cirrus components are shown in Fig. 4 and are much smaller than the signal and have different energy spectrum than these foregrounds, although we find that Channel $4(8 \mu \mathrm{m})$ data may contain a significant cirrus contribution. The latter cannot account for all the signal since 
the maps common to all four channels have statistically significant correlation and the cirrus emission is negligible at shorter wavelengths.

Contributions from ordinary galaxies come in two flavours: 1) the shot noise, and 2) due to clustering out of the primordial density fields which these galaxies trace. The shot-noise contribution, when evaluated directly from galaxy counts, gives a good fit to the fluctuations on smaller scales. Ordinary galaxies have been eliminated from the maps down to very faint flux levels $(\sim 0.3 \mu \mathrm{Jy}$ in Channel 1). The remaining galaxies' contribution to the CIB mean levels is small $\left(<0.1-0.2 \mathrm{nW} / \mathrm{m}^{2} / \mathrm{sr}\right)$ and in (13) we showed that their clustering component is much smaller than the signal in Fig. 4. On the other hand, the amplitude, power spectrum and the spectral energy dependence of the $>$ arcminute-scale fluctuation can be explained by emissions from Population III (13).

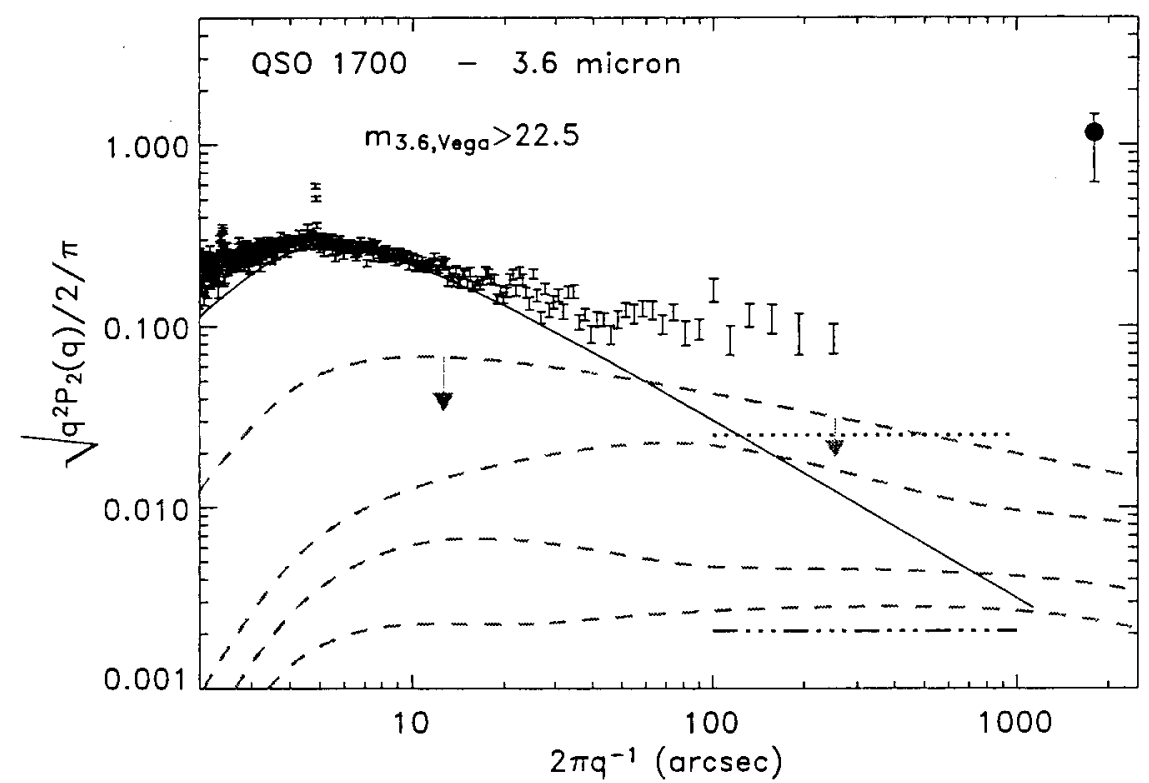

Figure 4 The spectrum of CIB fluctuations in the IRAC Channel 1 is shown with error bars. Solid line shows the shot noise contribution from ordinary galaxies. Contributions from the clustering component are shown with dashed lines, but are small: the top dashed line shows the upper limit assuming that galaxies at early times remained clustered as today, and the other dashed lines correspond to the remaining galaxies with flux less $0.3 \mu \mathrm{Jy}$ lying at $z \geq 1,3$ and 5 respectively. Cirrus and zodiacal emission contributions to the fluctuations are shown with dotted and dashed-dot-dot-dot lines respectively. The result at a larger scale from the DIRBE analysis (15), which includes contributions from all galaxies, is shown as filled circle with its $92 \%$ confidence level. 
The results are easier to interpret in terms of the high- $z$ Universe than the earlier DIRBE, deep 2MASS and NIRS data analyses discussed in Sec. 4. The latter claimed significant CIB levels in the NIR, both the mean levels $(6 ; 20 ; 29 ; 10 ; 5)$ and anisotropies $(15 ; 20 ; 16 ; 22)$, which are higher than the contributions from the observed ordinary galaxy populations, which saturate with the fainter galaxies contributing little to the total flux. However, any direct comparison between the old fluctuation measurements and the present detections is difficult because of the different range of angular scales, wavelengths, and because in the DIRBE (15) and NIRS analyses (20) no foreground galaxies were removed, and the deep 2MASS data also contained contributions from relatively bright galaxies. What is important is that the new measurement identifies directly the CIB fluctuations from very faint and, hence, distant sources and on scales $>1^{\prime}$ the fluctuations seem to be dominated by Population III emissions.

\section{Conclusions}

Cosmic infrared background often presents the only way to detect emissions from all cosmic times including from objects inaccessible to current or future telescopic studies. Because of the strong foreground emissions from the Galaxy and the Solar system, even in space-based measurements one often has to get at the CIB indirectly. On the other hand, in surveys with fine resolutions and good sensitivity one can remove galaxies down to sufficiently faint fluxes to start isolating contributions to the CIB and its structure from objects at progressively earlier times. If enough ordinary galaxies are removed, one can hope to see the contribution to the CIB from Population III era. We discussed the latest measurements of the CIB and its fluctuations and the constraints it set on early stellar populations. Our recent analysis of deep data from the Spitzer Space Telescope has allowed to remove galaxies to very faint levels of $\sim 0.3 \mu \mathrm{Jy}$. We find in the remaining data CIB anisotropies whose amplitude exceeds significantly that produced by the remaining galaxies and probably comes from the Population III epochs (13).

\section{Acknowledgements}

This work was supported by the NSF under Grant No. AST-0406587. I thank my collaborators on the Spitzer results presented in Sec. 5, Rick Arendt, John Mather and Harvey Moseley, for their contributions. 


\section{References}

[1] Arendt, R.G. \& Dwek, E. 2003, Ap.J., 585,305

[2] Arendt, R.G., Kashlinsky, A., Mather, J.C. \& Moseley, S.H. 2005, Ap.J., in preparation

[3] Blain, A. et al 2002, Physics Reports, 369, 111

[4] Cooray, A., Bock, J., Keating, B., Lange, A. \& Matsumoto, T. 2004, 606, 611

[5] Cambresy, L., Reach, W. T., Beichman, C.A. \& Jarrett, T. H. 2001, Ap.J., 555,563

[6] Dwek, E. \& Arendt, R. 1998, Ap.J.,508,L9

[7] Fazio, G. G. et al 2004, Ap.J.Suppl., 154, 10

[8] Fixsen, D. et al. 1998, Ap.J.,508, 123

[9] Fixsen, D., Moseley, S.H. \& Arendt, R.G. 2000, Ap.J. Suppl., 128, 651

[10] Gorjian, V., Wright, E.L., \& Chary, R.R. 2001,Ap.J.,536,550

[11] Hauser, M. et al. 1998, Ap.J., 508, 25

[12] Kashlinsky, A. 2005, Physics Reports, 409, 361

[13] Kashlinsky, A., Arendt, R.G., Mather, J.C. \& Moseley, S.H. 2005, Nature, in press

[14] Kashlinsky, A., Mather, J. \& Odenwald, S. 1996, Ap.J., 479, L9

[15] Kashlinsky, A. \& Odenwald, S. 2000a, Ap.J.,528,74

[16] Kashlinsky, A., Odenwald, S., Mather, J.C., Skrutskie, M. \& Cutri, R. 2002, Ap.J,579,L53

[17] Kashlinsky, A., Arendt, R., Gardner, J.P., Mather, J. \& Moseley, S.H. 2004, Ap.J., 608, 1

[18] Kelsall, T. et al 1998, Ap.J., 508,44

[19] Madau, P. \& Pozzetti, L. 2000,MNRAS,312,L9

[20] Matsumoto, T. et al 2005, Ap.J., 626, 31

[21] Metcalfe, L. et al. 2003, Astron. Astrophys., 407, 791

[22] Odenwald, S., Kashlinsky, A., Mather, J.C., Skrutskie, M. \& Cutri, R. 2003, Ap.J,583,535

[23] Papovich, C. et al. 2004, Ap.J. (Suppl.), 154, 70

[24] Puget, J.-L. et al. 1996, Astron. Astrophys., 308, L5

[25] Salvaterra, R. \& Ferrara, A. 2003, MNRAS, 339, 973

[26] Santos, M., Bromm, V. \& Kamionkowski, M. 2002, MNRAS, 336, 1082

[27] Schlegel, D., Finkbeiner, D. P. \& Davis, M. 1998, Ap.J., 500, 525

[28] Stanev, T. \& Franceschini, A. 1998, Ap.J., 494, L159

[29] Wright, E.L. \& Reese, E.D. 2000, Ap.J.,545,43 